\title{
Relación entre consultas a urgencias por enfermedad respiratoria y contaminación atmosférica en Ciudad J uárez, Chihuahua
}

\author{
Leticia Hernández-C adena, M. en $C$., ${ }^{(1)}$ Martha Ma.Téllez-Rojo, Mat., M. en $C$., ${ }^{(1)}$ \\ Luz Helena Sanín-A guirre, M. en C., D r. en C., ${ }^{(1,2)}$ Marina Lacasaña-N avarro, M. en C ., D r. en C., ${ }^{(1)}$ \\ Armando Campos, M. en C., ${ }^{(3)}$ Isabelle Romieu, M.D., M.P.H., Sc.D. ${ }^{(3,4)}$
}

\section{Hernández-Cadena L, Téllez-Rojo MM, Sanín-A guirre LH, Lacasaña-Navarro M, Campos A, Romieu I. \\ Relación entre consultas a urgencias por enfermedad respiratoria y contaminación atmosférica en Ciudad Juárez, Chihuahua. \\ Salud Publica Mex 2000;42:288-297.}

\section{Resumen}

Objetivo. Analizar la relación entre las concentraciones ambientales de partículas de diámetro $\leq 10 \mu \mathrm{m}\left(\mathrm{PM}_{10}\right)$ y de ozono con el numero diario de consultas al servicio de urgencias por enfermedades respiratorias agudas y asma en niños menores de 15 años, residentes de Ciudad Juárez, C hihuahua, México. Material y métodos. Este estudio se realizó durante los años de 1998 y 1999, utilizando un diseño de tipo ecológico. Los datos atmosféricos se obtuvieron de la base de datos de laA gencia de Protección al A mbiente (EPA), provenientes de ocho estaciones de monitoreo ubicadas en Ciudad Juárez y en El Paso,Texas. Los datos de consultas al servicio de urgencias por causas respiratorias se obtuvieron de los registros médicos de dos hospitales del Instituto Mexicano del Seguro Social (IMSS), en Ciudad Juárez, Chihuahua, de julio de 1997 a diciembre de 1998. Los diagnósticos se clasificaron en dos grupos: a) asma, y b) infecciones respiratorias altas, conforme a la Clasificación Internacional de Enfermedades (CIE-9 o CIE-10). El análisis se realizó con la utilización de la metodología de series de tiempo que emplea regresión Poisson. Resultados Durante el periodo de estudio la media de 24 horas de $P M_{1}$ fue de $34.46 \mu \mathrm{g} / \mathrm{m}^{3}$ (DE=17.99) y la media de los niveles de

\author{
Hernández-Cadena L, Téllez-Rojo MM, \\ Sanín-A guirre LH, Lacasaña-N avarro M, \\ Campos A, Romieu I. \\ Relationship between emergency room visits \\ for respiratory disease and atmospheric pollution \\ in Ciudad Juárez, Chihuahua. \\ Salud Publica Mex 2000;42:288-297.
}

\begin{abstract}
A bstract
Objective To assess the relationship of $\leq 10 \mu \mathrm{m}$ particles $\left(\mathrm{PM}_{10}\right)$ and atmospheric ozone concentrations, with the daily number of emergency visits due to asthma and acute respiratory diseases, among children aged under 15 , living in Ciudad Juarez, Chihuahua, Mexico. Material and methods. Between 1998 and 1999, an ecologic study was conducted. Atmospheric data were obtained from the Environmental ProtectionA gency (EPA), from eight monitoring stations located in Ciudad Juarez, Chihuahua, and El Paso, Texas. From July 1997 to D ecember 1998, data from emergency room visits for respiratory illness were abstracted from existing medical records of two Mexican Institute of Social Security (IMSS) hospitals in Ciudad Juarez. Diagnoses were classified into two groups: a) asthma, and b) upper respiratory infections (URI), according to the International Classification of D iseases (ICD -9 and/or ID C -10). Statistical analysis was carried out using the Poisson regression time series method. Results. During the study period, the mean 24-hour PM 1 level was $34.46 \mu \mathrm{g} / \mathrm{m}^{3}(S D=17.99)$ and the mean ozone level was $51.60 \mathrm{ppb}(S \mathrm{SD}=20.70)$. The model shows that an increase of $20 \mu \mathrm{g} / \mathrm{m}^{3}$ in the mean 24-hour exposure to $\mathrm{PM}_{10}$ was related to an increase of $4.97 \%(95 \%$
\end{abstract}

Este estudio tuvo el apoyo del Instituto Mexicano del Seguro Social y del $\mathrm{N}$ ational Center for Environmental Health/C enters for Disease Control and Prevention, Atlanta (GA), Estados Unidos de América (EUA).

(1) Centro de Investigación en Salud Poblacional, Instituto N acional de Salud Pública, Morelos, México. Becaria del Centro de Control y Prevención de Enfermedades/O rganización Panamericana de la Salud, Atlanta (GA), EUA.

(2) Facultad de Enfermería y N utrición, Universidad Autónoma de Chihuahua, Chihuahua, México.

(3) Instituto Mexicano del Seguro Social, Chihuahua, México.

(4) O rganización Panamericana de la Salud/Centro de Control y Prevención de Enfermedades,Atlanta (GA), EUA.

Fecha de recibido: 3 de enero de 2000 - Fecha de aprobado: 9 de junio de 2000

Solicitud de sobretiros: Mtra. Leticia Hernández Cadena, Centro de Investigación en Salud Poblacional, Instituto N acional de Salud Pública. Avenida Universidad 655, colonia Santa María A huacatitlán, 62508 Cuernavaca, Morelos, México.

Correo electrónico: Ihcadena@ insp3.insp.mx 
ozono fue de 51.60 partes por billón (ppb) $(D E=20.70)$. El modelo propuesto estima que un incremento de $20 \mu \mathrm{g} / \mathrm{m}^{3}$ en el promedio de 24 horas, en la exposición a PM relaciona con un incremento de $4.97 \%$ (IC $95 \%$ 0.97-9.13) en las consultas por asma con un retraso de cinco días y con un incremento de 9\% (IC 95\% 1.8-16.8) cuando se considera a la exposición acumulada de cinco días anteriores. Respecto a enfermedades respiratorias altas se encontró un aumento de $2.95 \%$ en las consultas a urgencias por cada $20 \mu \mathrm{m} / \mathrm{m}^{3}$ de incremento en el promedio de 24 horas en la exposición a $\mathrm{PM}_{10}$. Se observó que el impacto de $\mathrm{PM}_{10}$ sobre las visitas de urgencia por asma fue más severo en los días en que los niveles de ozono excedían los 49 ppb (nivel de la mediana) en el ambiente. Conclusiones Se encontraron asociaciones positivas entre las concentraciones de $\mathrm{PM}_{10}$ y el número de consultas por asma y enfermedades respiratorias aun cuando los niveles alcanzados no excedían las normas ambientales mexicanas. Asimismo, se detectó un efecto sinérgico entre ozono y $\mathrm{PM}_{10}$.

Palabras clave: enfermedades respiratorias; contaminantes ambientales del aire; partículas suspendidas; ozono; México
$\mathrm{Cl}$ 0.97-9.13) in emergency visits for asthma, with a 5-day lag, as well as to an increase of $9 \%(95 \% \mathrm{Cl} 1.8-16.8)$ when a cumulative 5-day exposure was considered. URI increased $2.95 \%$ as a cause of emergency room visits, for each 20 $\mu \mathrm{g} / \mathrm{m}^{3}$ increase in the mean 24-hour exposure to $\mathrm{PM}_{10}$. The impact of $\mathrm{PM}_{10}$ on emergency visits for asthma was greater on days with ${ }^{10}$ zone ambient levels exceeded 49 ppb (median value). Conclusions. A positive association was found between environmental PM ${ }_{10}$ and ozone concentrations and the daily number of emergency room visits due to asthma and acute respiratory diseases, even with levels lower than the Mexican standard levels. Also, a synergic effect between $\mathrm{PM}_{10}$ and $\mathrm{O}_{3}$ was found.

Key words: respiratory tract diseases; air pollutants, environmental; suspended particles; ozone; Mexico
C iudad Juárez, Chihuahua, es una de las ciudades de la República mexicana que forman parte de la franja fronteriza con Estados Unidos de América (EUA), que se conoce como región Paso del Norte. Esta zona presenta condiciones climáticas y geográficas propicias para que, de no tomarse medidas de control oportunas, en un futuro se generen serios problemas de contaminación atmosférica. Ciudad Juárez tiene un clima semiárido y se encuentra rodeada por importantes cadenas montañosas que impiden la libre circulación del aire. Al igual que otras comunidades de la misma franja fronteriza, esta ciudad ha experimentado una acelerada expansión demográfica y un importante incremento en su actividad económica a lo largo de las últimas décadas; aunado a ello, se han desarrollado un sinnúmero de actividades tanto industriales como comerciales y de servicios. Estas condiciones han provocado una degradación de la calidad del aire, especialmente por la utilización de vehículos en mal estado, así como por el crecimiento del parque vehicular, que asciende a 366739 vehículos, los cuales constituyen la principal fuente de contaminación en dicha ciudad. Según informes de la Dirección General de Finanzas del Gobierno del Estado de Chihuahua, de 1997, $88 \%$ del total de contaminantes emitidos a la atmósfera provienen de vehículos que circulan en Ciudad Juárez. ${ }^{1}$

En los meses de otoño e invierno, dadas las condiciones geográficas de la ciudad, el aire caliente forma una capa sobre las masas de aire frío cargadas de con- taminantes, manteniendo a éstos a nivel de la superficie de la tierra e impidiendo que se dispersen, ${ }^{2, *}$ lo que ocasiona un fenómeno de inversión térmica que, además, acrecienta los problemas de contaminación en dicha ciudad.

Estudios epidemiológicos previos, hechos en México $^{3}$ y otros países, ${ }^{4}$ han analizado los efectos de la contaminación del aire, $\mathrm{y}$ han encontrado asociaciones entre los efectos agudos de enfermedad respiratoria en la población general y la exposición a contaminantes atmosféricos. Algunos estudios utilizan mediciones indirectas de efectos, como son el ausentismo escolar, ${ }^{5,6}$ o la demanda de servicios hospitalarios o de urgencias. ${ }^{7-10}$ Adicionalmente, se miden condiciones ambientales y meteorológicas (velocidad y dirección del viento, humedad relativa y temperatura) que se consideran como confusores potenciales en el análisis.

La mayor parte de los estudios sobre asociaciones entre la exposición a contaminantes atmosféricos y el número de consultas por enfermedades respiratorias se han realizado en lugares que presentan concentraciones de contaminación elevadas. ${ }^{711,12}$ En la ciudad de México se han hecho algunos estudios con la metodología de series de tiempo; sin embargo, las condiciones atmosféricas son muy diferentes a las de Ciudad Juá-

* Richards AW. Report from Governor's Border Working Group, 1993. 
rez, ya que se han presentado niveles de contaminación de hasta $378 \mu \mathrm{g} / \mathrm{m}^{3}$ de partículas de diámetro menor o igual a $10 \mu \mathrm{m}\left(\mathrm{PM}_{10}\right)$ y 404 partes por billón (ppb) de ozono $\left(\mathrm{O}_{3}\right){ }_{1}^{13}$ las cuales rebasan la norma la mayor parte del año. No obstante, los pocos estudios que se han realizado en otros lugares del mundo con bajos niveles de contaminantes han encontrado una asociación positiva. $^{14}$

En el cuadro I se muestran los riesgos relativos de consultas al servicio de urgencias por asma encontrados en estudios hechos durante los últimos cinco años, que persiguen objetivos similares y emplean la misma metodología que el presente. Los riesgos relativos fueron estandarizados para un incremento común (20 ppb de $\mathrm{O}_{3 \mathrm{y}} 20 \mu \mathrm{g} / \mathrm{m}^{3}$ de $\mathrm{PM}_{10}$ ) con el objeto de hacerlos comparables. $., 7,11,12,15-20$

El objetivo de este estudio fue analizar, mediante la metodología de series de tiempo, la variación diaria de consultas a urgencias por enfermedades respiratorias agudas (asma y enfermedades respiratorias altas) de dos hospitales de concentración de segundo nivel del Instituto Mexicano del Seguro Social (IMSS), respecto a la variación diaria de los niveles de contaminación del aire en Ciudad Juárez, con la hipótesis de que existe una asociación positiva entre ellos.

\section{Material y métodos}

Se seleccionaron las dos unidades existentes de atención hospitalaria para población derechohabiente del IMSS en la región de estudio, las que atienden aproximadamente a $60 \%$ de la población y cuentan con servicio de urgencias. Estas unidades son, además, las que mayor número de casos de enfermedades respiratorias notifican en dicha región. Se tomó en cuenta el número total de consultas de primera vez por alguna enfermedad respiratoria aguda de niños menores de 15 años de edad dentro del servicio de urgencias de los dos hospitales referidos, durante el periodo comprendido entre el 1 julio de 1997 y el 25 de diciembre de 1998 (543 días).

Los casos se identificaron con el empleo del diagnóstico médico consignado en los registros de ingresos de urgencias (hojas 4-30-6). Para cada caso se obtuvo fecha y turno (matutino/vespertino) en que se solicitó la consulta, información sobre tipo de consulta (primera vez o subsecuente), edad del paciente y diagnóstico principal.

Los casos se identificaron de acuerdo con la Clasificación Internacional de Enfermedades, utilizando la novena revisión (CIE-9) ${ }^{21}$ para los datos registrados en las fechas de julio de 1997 a octubre de 1997 y la décima revisión (CIE-10) ${ }^{22}$ para datos registrados de noviembre de 1997 a diciembre de 1998. Las consultas por padecimientos respiratorios se clasificaron en dos grupos: a) de asma (CIE-9: 493, CIE-10: J45), y b) de infecciones respiratorias altas (IRA). Este segundo grupo quedó integrado por los siguientes padecimientos: infecciones respiratorias agudas no especificadas (CIE-9: 460, CIE-10: J06.9); sinusitis aguda (CIE-9: 461, CIE-10: J01.9); faringitis aguda (CIE-9: 462, CIE-10: J02.9); laringitis, traqueítis (CIE-9: 464, CIE10: J04x y CIE-10: J06.0), y tos (CIE-9: 463).

Los datos de salud se capturaron mediante el paquete del Sistema de información médica y operativa (SIMO) que se utiliza en la captura rutinaria que rea-

Cuadro I

Riesgos relativos para consultas de URGencias por ASMA EN DIFERENTES ESTUdios

\begin{tabular}{|c|c|c|c|c|c|c|}
\hline \multirow[b]{2}{*}{ Autor } & \multirow[b]{2}{*}{ Población de estudio } & \multirow[b]{2}{*}{ Edad (años) } & \multicolumn{2}{|c|}{$\mathrm{O}_{3}(\mathrm{ppb})$} & \multicolumn{2}{|c|}{$\mathrm{PM}_{10}\left(\mu \mathrm{g} / \mathrm{m}^{3}\right)$} \\
\hline & & & Media & $\mathrm{RR}^{*}$ & Media & $\mathrm{RR}^{\ddagger}$ \\
\hline W hite $M$ & Atlanta, EUA $(1990)^{11}$ & 1 a 16 & 78 & - & 30.9 & 1.0404 \\
\hline Atkinson RW & Londres, RU (1992-1994) & - & - & - & - & 1.0193 \\
\hline Delfino RJ & Montreal,Q uebec,C anadá (1984-1998)16 & - & - & 1.0454 & - & - \\
\hline Clifford P & N ueva Jersey, C anadá (1988-1989)17 & Todas las edades & 48.0 & 1.1300 & - & NS \\
\hline Stieb DM & St. John, N ew Brunswick, C anadá (1989-1992) ${ }^{18}$ & Todas las edades & 41.6 & 1.0790 & - & - \\
\hline Lipsett M & Santa Clara C ounty, California, EUA (1988-1989/1991-1992) ${ }^{19}$ & & - & - & - & 1.1266 \\
\hline Schwartz J & Seattle,W ashington, EUA (1989-1990) ${ }^{6}$ & $<65$ & - & Ns & 29.6 & 1.0754 \\
\hline Tenias JM & Valencia, España (1994-1995) ${ }^{12}$ & Todas las edades & 62.8 & 1.1300 & - & - \\
\hline Romieu I & México, D.F., México $(1990)^{7}$ & $<16$ & 90.0 & 1.1538 & - & - \\
\hline Téllez-Rojo MM & México, D.F., México $(1995)^{20}$ & $<15$ & & 1.1107 & & - \\
\hline \multicolumn{2}{|c|}{$\begin{array}{l}\text { N S: no significativo } \\
* \text { Riesgo relativo estimado para un incremento de } 20 \mathrm{ppb} \text { de } 0_{3} \\
\text { ₹ Riesgo relativo estimado para un incremento de } 20 \mu \mathrm{g} / \mathrm{m}^{3} \text { de } \mathrm{PM}_{10}\end{array}$} & \multicolumn{3}{|c|}{$\begin{array}{l}\text { EUA: Estados Unidos de América } \\
\text { RU: Reino Unido }\end{array}$} & & \\
\hline
\end{tabular}


liza el IMSS. Para asegurar la calidad de los datos se tomó una muestra de los registros de ingreso y se midió la concordancia con los datos capturados, de tal forma que pudiera detectarse algún error en la captura. El resultado de esta revisión fue de $4.62 \%$ de error.

Los datos de contaminantes aéreos y condiciones meteorológicas, procedentes de la Agencia de Protección al Ambiente (EPA, por sus siglas en inglés), fueron proporcionados por la Universidad del Estado de Texas. Esta información proviene de monitores ubicados tanto en El Paso, Texas, como en Ciudad Juárez, Chihuahua, ya que aun cuando a estas ciudades las separa una línea fronteriza internacional, en la vida cotidiana forman una sola área metropolitana. Se registraron así los niveles de contaminación por $\mathrm{O}_{3}$ y por $\mathrm{PM}_{10}$; asimismo, se consideraron parámetros meteorológicos de temperatura, humedad relativa y velocidad del viento que son recabados en ocho estaciones de monitoreo, de las cuales siete hacen mediciones de $\mathrm{PM}_{10}$, cuatro de $\mathrm{O}_{3}$ y cuatro de condiciones meteorológicas. Del total de estas estaciones, dos se encuentran ubicadas en El Paso; sin embargo, se decidió tomarlas en cuenta por ser las más cercanas a Ciudad Juárez.

Se generó una base de datos secundaria a partir de los promedios diarios de los distintos monitores considerados para cada una de las variables. De esta forma, se generaron las medias diarias de concentraciones de $\mathrm{PM}_{10}$ en 24 horas, las medias diarias de $\mathrm{O}_{3}$ del promedio de seis horas de niveles máximos (de las 10 a las 16 horas) y del nivel horario máximo del día, además, el máximo, mínimo y media de temperatura, humedad relativa y velocidad del viento.

Se construyeron variables que asociaran los niveles de $\mathrm{PM}_{10} \mathrm{y} \mathrm{O}_{3}$ con las visitas al servicio de urgencias con un retraso de 1 a 7 días con el objeto de estudiar diferentes patrones de asociación. Para las variables meteorológicas se construyeron también variables con un día de retraso.

Se realizó un análisis descriptivo de todas las variables con el propósito de conocer su comportamiento. Se generó una variable indicadora para diferenciar los días de fin de semana de los de entre semana, y otra referida al año de estudio para controlar efectos de largo plazo; además, se generó una variable categórica para meses fríos (noviembre a marzo) y para meses cálidos (abril a octubre).

El porcentaje de datos faltantes sobre los contaminantes y variables meteorológicas fue inferior a $2 \%$ para $\mathrm{O}_{3}$ y a $11 \%$ para $\mathrm{PM}_{10}$. Estos valores se estimaron por medio de regresión lineal al utilizar como predictores las variables ambientales disponibles en el día correspondiente al dato faltante.
Se construyó un modelo básico para las consultas al servicio de urgencias por enfermedad respiratoria, que explicara la mayor variabilidad y ajustara por tendencias periódicas y estacionales. Este modelo quedó conformado por temperatura mínima y por variables indicadoras de fin de semana, de días festivos, año calendario y estación. No se dispuso de información acerca de la presentación de epidemias que pudieran ocasionar un aumento inesperado en el número de consultas al servicio de urgencias.

Para evaluar posibles periodos de latencia se estudió la relación entre el número de consultas por cada grupo de enfermedades y los niveles de contaminantes en los siete días previos a la consulta. Asimismo, el efecto acumulado de la exposición se evaluó utilizando la medición promedio de los contaminantes correspondiente a los tres, cinco y siete días consecutivos anteriores al evento. En tanto que para evaluar estas asociaciones se utilizó un modelo de regresión Poisson, ya que la variable dependiente es una variable de conteo que únicamente toma valores enteros no negativos y pequeños en relación con el tamaño de la población.

Para incorporar la estructura de autocorrelación derivada de observaciones seriadas se construyeron modelos de efectos fijos y aleatorios, ${ }^{23}$ tomando el año calendario como variable de grupo; no obstante, la prueba de hipótesis que compara esta técnica con una regresión Poisson estándar indicó que esta última era la más adecuada para el análisis de los datos por lo que se decidió utilizarla. Con el propósito de incorporar cualquier posible autocorrelación inherente a un estudio de series de tiempo se filtraron ${ }^{24}$ los datos a través de un promedio móvil del número de consultas de seis días, el que se incluyó en el modelo final. Al incorporar esta nueva variable aumentó significativamente el porcentaje de devianza explicada por el modelo.

Se exploraron posibles asociaciones no lineales entre cada una de las variables independientes continuas $\left(\mathrm{PM}_{10}, \mathrm{O}_{3}\right.$, temperatura, humedad y velocidad del viento) con la variable dependiente mediante los modelos aditivos generalizados, ${ }^{24,25}$ sin embargo, en ningún caso se rechazó la hipótesis de linealidad.

Los modelos propuestos se validaron con técnicas de diagnóstico (bondad de ajuste y análisis de influencia) basadas en los residuos estandarizados. Además, para dichos residuos se exploró la posible existencia de autocorrelación remanente, pero no se encontró ninguna significativa.

Para describir la asociación entre el número de consultas esperadas por asma y los niveles de $\mathrm{PM}_{10}$ 
se realizó un suavizamiento utilizando la técnica no paramétrica Lowess.

Los resultados obtenidos (riesgos relativos en las consultas) se calcularon para un incremento de 20 unidades de la variable de exposición y se interpretaron en términos porcentuales. El análisis de la información se desarrolló utilizando el paquete Stata versión 6.0 .

\section{Resultados}

Durante el periodo de estudio ocurrieron un total de 12721 consultas de niños menores de 15 años por IRA y 2549 por asma en los servicios de urgencias de los dos hospitales considerados. En el cuadro II se muestran las estadísticas descriptivas diarias. El mayor número de consultas por enfermedades respiratorias en niños se presentó en el grupo de 1 a 4 años, con aproximadamente $50 \%$ de los casos, seguido por el grupo de niños menores de un año. Los grupos de 5 a 9 y de 10 a 14 años presentaron menor número de demandas por enfermedades respiratorias (23 y $11.5 \%$, respectivamente).

En el cuadro II también se muestran las estadísticas descriptivas básicas de los niveles de contaminantes y variables meteorológicas. Se puede observar que la concentración promedio máxima de $\mathrm{PM}_{10} \mathrm{du}$ rante el periodo de estudio fue de $167.47 \mu \mathrm{g} / \mathrm{m}^{3}$, mientras que para $\mathrm{O}_{3}$ fue de $262 \mathrm{ppb}$. Sólo un día del periodo de estudio se rebasó la norma mexicana para $\mathrm{PM}_{10}$
(150 $\mu \mathrm{g} / \mathrm{m}^{3}$ para la media de 24 horas) y, en el caso de $\mathrm{O}_{3}$, la norma se rebasó durante cinco días (110 ppb para 1 hora máxima); esto ocurrió en el segundo semestre de 1998. Las correlaciones de los contaminantes entre los monitores utilizados para estimar la exposición media diaria fluctuaron entre 0.85 y $0.99(p<0.01)$ para $\mathrm{O}_{3}$, entre 0.32 y $0.91(p<0.05)$ para $\mathrm{PM}_{10}$.

Al aplicar la técnica de regresión Poisson, que asume riesgo constante, se encontró una asociación positiva significativa entre los niveles de $\mathrm{PM}_{10}$ y el número de consultas por asma para el periodo de latencia de cinco días; sin embargo, esta significancia estadística no se observó para los demás periodos de latencia considerados (cuadro III). Se observó que un incremento de $20 \mu \mathrm{g} / \mathrm{m}^{3}$ de $\mathrm{PM}_{10}$ (rango intercuartílico) en el promedio de 24 horas ocasionaría un incremento de $4.97 \%$ (RR 1.0497) en las consultas por asma con un retraso de cinco días (IC $95 \% 0.95$ - 9.13). Asimismo, se observó que la exposición acumulada a partículas tiene un mayor efecto sobre las consultas después de cinco días consecutivos; el aumento esperado en las consultas fue de $9.04 \%$ (RR 1.0904, IC 95\% 1.0177 - 1.1683). No se encontró asociación significativa entre los niveles de ozono y las consultas por asma; sin embargo, se obtuvo una asociación positiva, marginalmente significativa con un periodo de latencia de seis días (cuadro III).

Para las IRA (cuadro III) también se observó que un incremento de $20 \mu \mathrm{g} / \mathrm{m}^{3}$ de $\mathrm{PM}_{10}$ en el promedio de 24 horas podría tener un efecto inmediato e in-

\section{Cuadro II \\ Estadísticas descriptivas de las variables utilizadas en Ciudad Juárez, Chihuahua, DE JULIO DE 1997 A DICIEMBRE DE 1998}

\begin{tabular}{|c|c|c|c|c|c|c|c|c|}
\hline \multirow[b]{2}{*}{ Variable } & \multicolumn{2}{|c|}{ Rango } & \multirow[b]{2}{*}{ Media } & \multirow[b]{2}{*}{ DE } & \multicolumn{3}{|c|}{ Percentiles (\%) } & \multirow{2}{*}{$\begin{array}{c}\text { Proporción de atenciones } \\
\text { diarias }(\%)\end{array}$} \\
\hline & Mínimo & Máximo & & & 25 & 50 & 75 & \\
\hline Consultas diarias por asma & 0 & 18 & 4.70 & 3.36 & 2 & 4 & 7 & 20 \\
\hline Consultas diarias por IRA & 4 & 80 & 23.47 & 12.22 & 15 & 20 & 29 & 80 \\
\hline Total de consultas diarias & 6 & 87 & 28.17 & 12.88 & 19 & 26 & 35 & 100 \\
\hline
\end{tabular}

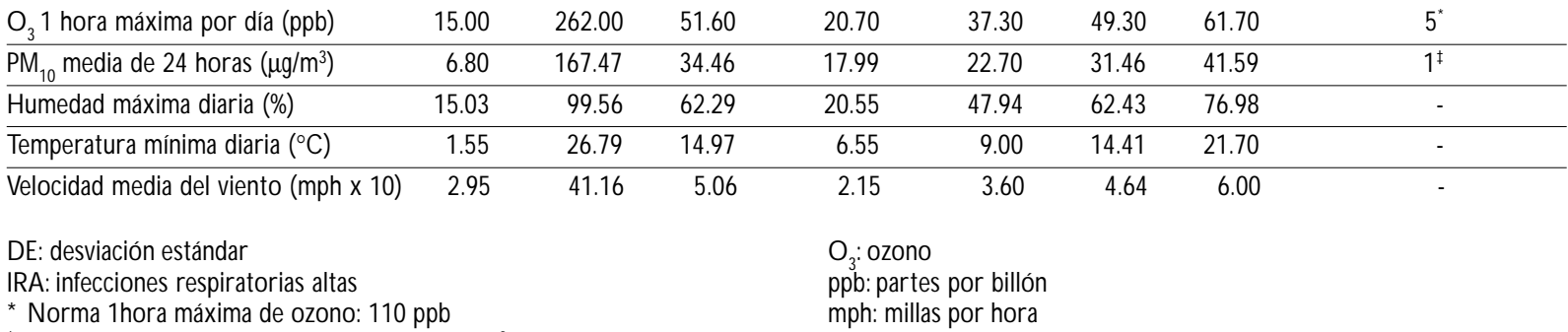

₹ Norma promedio de 24 horas $\mathrm{PM}_{10}: 150 \mu \mathrm{g} / \mathrm{m}^{3}$ 
crementar las consultas a urgencias en $2.95 \%$ (IC 95\% 0.8 - 5.07), después de tres días de la exposición a dicho contaminante. También se encontró que la exposición acumulada a partículas muestra un efecto mayor que la exposición por periodos de latencia; así, una exposición acumulada de siete días consecutivos provocaría un aumento de $4.51 \%$ (IC $95 \%$ 0.70 - 8.47) en las consultas $(p<0.05)$. Los niveles de ozono, por su parte, ocasionarían un aumento en las consultas por IRA de $2.96 \%$ (IC 95\% 0.91 - 5.05) un día después de haberse presentado un incremento de $20 \mathrm{ppb}$.

Se evaluó la asociación de la exposición a $\mathrm{O}_{3}$ y $\mathrm{PM}_{10}$ con infecciones respiratorias bajas (IRB) pero no se encontró un patrón de asociación.
Dado que en el ambiente los contaminantes no se encuentran aislados sino en forma de mezclas, se intentó construir un modelo que incluyera el efecto más importante de ambos contaminantes $\left(\mathrm{O}_{3}\right.$ y $\left.\mathrm{PM}_{10}\right)$ cuando se presentan simultáneamente, en donde encontramos que, pese a la posible multicolinealidad subyacente, ambos contaminantes mantienen su significancia y muestran, además, un aumento en el riesgo relativo. Mientras que el efecto estimado de $\mathrm{PM}_{10^{\prime}}$ sin la presencia de $\mathrm{O}_{3}$, era de $4.97 \%$ para un incremento de $20 \mu \mathrm{g} / \mathrm{m}^{3}$ cinco días después, ajustando por la presencia del $\mathrm{O}_{3^{\prime}}$ este riesgo aumenta a $9.4 \%$. Aunque el correspondiente a $\mathrm{O}_{3}$ no presentó significancia estadística, el riesgo en el retraso de tres días aumenta de 2.99 a $4.37 \%$. Al estratificar por días con niveles

\section{Cuadro III \\ Riesgos Relativos PARA CONSULtAS A URGENCIAS POR ASMA Y ENFERMEDADES RESPIRATORIAS ALTAS en RELACIÓN CON LOS Niveles de OZONo $\left(0_{3}\right)$ Y PM 10 EN La atmósfera. Ciudad Juárez, Chinuahua, de julio 1997 a diciembre de 1998}

\begin{tabular}{|c|c|c|c|c|c|c|}
\hline \multirow{3}{*}{$\begin{array}{l}\mathrm{O}_{3}(\mathrm{ppb}) \\
1 \text { día anterior }\end{array}$} & \multicolumn{3}{|c|}{ Asma } & \multicolumn{3}{|c|}{ IRA } \\
\hline & \multirow{2}{*}{$\begin{array}{c}R^{*} \\
1.0222\end{array}$} & \multicolumn{2}{|c|}{ IC 95\% } & \multirow{2}{*}{$\begin{array}{c}\mathrm{RR}^{*} \\
1.0296\end{array}$} & \multicolumn{2}{|c|}{ IC $95 \%$} \\
\hline & & 0.9821 & 1.0640 & & 1.0091 & 1.0505 \\
\hline 2 días anteriores & 1.0053 & 0.9613 & 1.0514 & 0.9706 & 0.9504 & 0.9912 \\
\hline 3 días anteriores & 1.0299 & 0.9865 & 1.0752 & 1.0024 & 0.9830 & 1.0221 \\
\hline 4 días anteriores & 1.0009 & 0.9581 & 1.0456 & 0.9847 & 0.9639 & 1.0061 \\
\hline 5 días anteriores & 0.9936 & 0.9510 & 1.0381 & 0.9919 & 0.9707 & 1.0137 \\
\hline 6 días anteriores & 1.0347 & 0.9954 & 1.0757 & 0.9976 & 0.9764 & 1.0192 \\
\hline 7 días anteriores & 0.9880 & 0.9462 & 1.0316 & 0.9903 & 0.9690 & 1.0120 \\
\hline Promedio 3 días & 1.0405 & 0.9787 & 1.1061 & 1.0007 & 0.9720 & 1.0301 \\
\hline Promedio 5 días & 1.0337 & 0.9573 & 1.1161 & 0.9868 & 0.9511 & 1.0238 \\
\hline Promedio 7 días & 1.0470 & 0.9595 & 1.1424 & 0.9811 & 0.9409 & 1.023 \\
\hline $\mathrm{PM}_{10}\left(\mu \mathrm{g} / \mathrm{m}^{3}\right)$ & $\mathrm{RR}^{\ddagger}$ & & & $\mathrm{RR}^{\ddagger}$ & & \\
\hline 1 día anterior & 1.0251 & 0.9858 & 1.0659 & 1.0185 & 0.9989 & 1.0385 \\
\hline 2 días anteriores & 1.0350 & 0.9911 & 1.0807 & 1.0082 & 0.9871 & 1.0297 \\
\hline 3 días anteriores & 1.0212 & 0.9783 & 1.0661 & 1.0295 & 1.0087 & 1.0507 \\
\hline 4 días anteriores & 1.0241 & 0.9849 & 1.0648 & 0.9923 & 0.9732 & 1.0118 \\
\hline 5 días anteriores & 1.0497 & 1.0097 & 1.0913 & 1.0115 & 0.9929 & 1.0305 \\
\hline 6 días anteriores & 0.9858 & 0.9464 & 1.0269 & 1.0162 & 0.9976 & 1.0350 \\
\hline 7 días anteriores & 0.9761 & 0.9346 & 1.0194 & 1.0153 & 0.9947 & 1.0363 \\
\hline Promedio 3 días & 1.0492 & 0.9910 & 1.1109 & 1.0341 & 1.0057 & 1.0633 \\
\hline Promedio 5 días & 1.0904 & 1.0177 & 1.1683 & 1.0306 & 0.9974 & 1.0650 \\
\hline Promedio 7 días & 1.0634 & 0.9824 & 1.1511 & 1.0451 & 1.0070 & 1.0847 \\
\hline
\end{tabular}

N ota: los riesgos relativos están ajustados por autocorrelación de las consultas (promedio acumulado de seis días), temperatura mínima, fin de semana, días festivos, indicador del año y estación

* RR: riesgo relativo estimado por un incremento equivalente al rango intercuartil: $(20 \mathrm{ppb}) \mathrm{RR}=\exp \left(\beta^{*} 20 \mathrm{ppb}\right)$

₹ RR: riesgo relativo estimado por un incremento equivalente al rango intercuartil: $\left(20 \mu \mathrm{g} / \mathrm{m}^{3}\right) \mathrm{RR}=\exp \left(\beta^{*} 20 \mu \mathrm{g} / \mathrm{m}^{3}\right)$

IRA: infecciones respiratorias altas 
altos de ozono (utilizando como punto de corte la mediana observada de $49 \mathrm{ppb}$ ) se encontró un riesgo relativo para asma de 1.1025 (IC 95\% 1.022 - 1.189) por cada aumento de $20 \mu \mathrm{g} / \mathrm{m}^{3}$ en los niveles de $\mathrm{PM}_{10}$ con retraso de un día. Este efecto disminuye conforme aumenta el periodo de latencia; sin embargo, el efecto de $\mathrm{PM}_{10}$ en días con niveles bajos de $\mathrm{O}_{3}$ no muestra significancia estadística sino hasta después de cinco días de exposición. Esto sugiere un posible efecto sinérgico entre estos contaminantes (cuadro IV).

En general, estos resultados indican que las condiciones que favorecen el desarrollo de síntomas de asma que ocasionarían un aumento en las visitas a urgencias se presentan en las épocas del año en las que la temperatura es baja y las concentraciones de $\mathrm{PM}_{10} \mathrm{y}$ $\mathrm{O}_{3}$ aumentan.

\section{Discusión}

Este estudio se realizó con el propósito de complementar los resultados obtenidos en una investigación binacional acerca de los efectos de la calidad del aire sobre la salud en la región fronteriza entre EUA y México.* Este estudio se llevó a cabo, inicialmente, en El Paso, Texas, y Las Cruces, Nuevo México, EUA, entre junio de 1994 y diciembre de 1995, en una población de niños menores de 17 años. De acuerdo con los resul-

* Hart R, VanDerslice J, Vera B, Gotway-Crawford C, Kieszak S, Phien $\mathrm{R}$ et al. Ambient air quality and acute pediatric illness in the Paso del Norte Airshed. CDC final report. May 1999. tados obtenidos en dicho estudio, un aumento de $20 \mu \mathrm{g} / \mathrm{m}^{3}$ en los niveles de $\mathrm{PM}_{10}$ ocasionaría un incremento de $6.7 \%$ en las consultas diarias a urgencias por asma, con un periodo de latencia de dos días. En el presente estudio se estimó que el incremento correspondiente sería de $4.97 \%$, pero con un periodo de latencia de cinco días. Estos resultados difieren un poco de los encontrados en el trabajo realizado en El Paso y en otros estudios, probablemente por las diferencias en el tiempo que tardan los individuos en solicitar los servicios de salud y la severidad de los casos recibidos. En Chile, un estudio realizado por Illabaca y colaboradores ${ }^{26}$ documenta resultados semejantes a los de la presente investigación en relación con el uso de servicios de salud. Sin embargo, la dirección de la asociación del estudio realizado en El Paso y la presente investigación es positiva respecto al incremento en los niveles de $\mathrm{PM}_{10} \mathrm{y}$ las consultas a urgencias por asma.

Los presentes resultados muestran consistencia en relación con lo obtenido en otros estudios sobre consultas a urgencias que han utilizado la misma metodología en diferentes partes del mundo. Investigaciones hechas en St John, New Brunswick, Canadá, y Nueva Jersey, EUA, donde la concentración media observada osciló entre 40 y $50 \mathrm{ppb}$ de ozono, han presentado riesgos de 7 y 13\%, respectivamente, de incremento en consultas por asma por cada aumento de $20 \mathrm{ppb}$ en los niveles de $\mathrm{O}_{3}{ }^{17,18}$ aunque otros estudios que han considerado niveles más altos de ozono han presentado riesgos menores. ${ }^{16}$ Para $\mathrm{PM}_{10}$ a pesar de que son limitadas las investigaciones que han notificado una asociación con visitas a urgencias, encontramos ma-

\section{Cuadro IV \\ Riesgos relativos para consultas a URgencias por asma en relación con los niveles de PM 10 estratificados por niveles de ozono del mismo día. Ciudad Juárez, Chihuahua, de Julio de 1997 a diciembre de 1998}

\begin{tabular}{|c|c|c|c|c|c|c|}
\hline \multirow{3}{*}{$\begin{array}{l}\mathrm{PM}_{10} \\
1 \text { día anterior }\end{array}$} & \multicolumn{3}{|c|}{$\mathrm{O}_{3} \leq 49 \mathrm{ppb}^{*}$} & \multicolumn{3}{|c|}{$\mathrm{O}_{3}>49 \mathrm{ppb} *$} \\
\hline & \multirow{2}{*}{$\begin{array}{c}\text { RR } \\
0.9921\end{array}$} & \multicolumn{2}{|c|}{ IC $95 \%$} & \multirow{2}{*}{$\begin{array}{c}\text { RR } \\
1.1025\end{array}$} & \multicolumn{2}{|c|}{ IC $95 \%$} \\
\hline & & 0.9454 & 1.0410 & & 1.0218 & 1.1896 \\
\hline 2 días anteriores & 1.0319 & 0.9789 & 1.0877 & 1.0523 & 0.9712 & 1.1401 \\
\hline 3 días anteriores & 1.0095 & 0.9574 & 1.0644 & 1.0392 & 0.9603 & 1.1245 \\
\hline 4 días anteriores & 1.0286 & 0.9833 & 1.0760 & 1.0097 & 0.9304 & 1.0958 \\
\hline 5 días anteriores & 1.0752 & 1.0289 & 1.1236 & 0.9662 & 0.8907 & 1.0482 \\
\hline 6 días anteriores & 1.0031 & 0.9564 & 1.0521 & 0.9318 & 0.8581 & 1.0119 \\
\hline 7 días anteriores & 0.9506 & 0.9009 & 1.0031 & 1.0771 & 0.9925 & 1.1688 \\
\hline
\end{tabular}


yor consistencia. El estudio realizado por White, ${ }^{11}$ en Atlanta en 1990, notifica un riesgo de $4.04 \%$ en las consultas por cada $20 \mu \mathrm{g} / \mathrm{m}^{3}$ de incremento de $\mathrm{PM}_{10^{\prime}}$ cuando los niveles de este contaminante tienen una media de $30.9 \mu \mathrm{g} / \mathrm{m}^{3}$, semejante a la de los observados en Ciudad Juárez durante el periodo de estudio (media de $34.46 \mu \mathrm{g} / \mathrm{m}^{3}$ ); o bien, también se puede mencionar el estudio realizado por Schwartz, ${ }^{6}$ en Seattle, Washington, que presenta un incremento de $7.5 \%$, con una media de $\mathrm{PM}_{10}$ de $29.6 \mu \mathrm{g} / \mathrm{m}^{3}$ (cuadro I).

En la ciudad de México los estudios sobre consultas a urgencias son muy pocos; el estudio realizado por Téllez-Rojo y colaboradores, ${ }^{20}$ en 1995, en niños menores de 15 años, sostiene que por cada 50 ppb de incremento en la concentración horaria máxima de ozono se ocasionaría un aumento en las visitas a urgencias por IRA de $9.9 \%$ en el día posterior y de hasta $30 \%$ al quinto día de exposición acumulada a tal contaminante. Sin embargo, en este estudio las mediciones para $\mathrm{PM}_{10}$ se hicieron cada seis días por lo que no se estudiaron periodos de latencia de $\mathrm{PM}_{10}$ ni se presentan riesgos relativos. Romieu y colaboradores ${ }^{7}$ han descrito que un incremento de $50 \mathrm{ppb}$ de ozono se asocia con $43 \%$ de incremento en el número de visitas a urgencias por asma con un día de retraso y de hasta $133 \%$ con retraso de dos días cuando los niveles de ozono exceden de las $110 \mathrm{ppb}$. Dicho estudio presenta la limitante de que se realizó en un periodo corto (incluye seis meses únicamente) y, dado que sólo cuenta con 44 mediciones de $\mathrm{PM}_{10}$, no presenta la estimación del efecto asociado a este contaminante. En este estudio no se informan riesgos relativos a más de dos días de retraso.

Por otro lado, algunos estudios muestran que las $\mathrm{PM}_{10}$ están relacionadas con problemas respiratorios agudos, ${ }^{7}$ Montealegre y colaboradore ${ }^{27}$ analizaron registros de hospitales en Puerto Rico, de 1987 a 1989, y encontraron una correlación entre admisión hospitalaria por crisis de asma y los niveles de $\mathrm{PM}_{10}$. Estudios más recientes que utilizan diseños transversales han encontrado riesgos relativos de hasta 1.4 para exacerbación de asma en niños asociados con contaminación por $\mathrm{PM}_{10}{ }^{28,29}$ Incluso se ha relacionado a las partículas con problemas de mortalidad por enfermedades respiratorias. $^{30}$

Todos estos trabajos sugieren una asociación entre niveles de contaminantes del aire y problemas respiratorios como bronquitis crónica, asma bronquial, enfisema pulmonar, rinitis, sinusitis y otras enfermedades agudas.

El presente estudio encontró que la exposición acumulada a partículas de tamaño menor a $10 \mu \mathrm{m}$ después de cinco días producen un efecto estimado sobre las visitas a urgencias por asma. El aumento en las consultas por IRA es, sin embargo, más significativa después de tres días de la exposición a $\mathrm{PM}_{10}$.

Además se detectaron efectos sobre la salud respiratoria (asma y enfermedades respiratorias altas) aun con niveles bajos de $\mathrm{PM}_{10}$, ya que sólo durante cinco días, dentro del periodo que abarca la investigación, se rebasó la norma para este contaminante. Aunque Ciudad Juárez no presenta graves problemas de contaminación atmosférica es recomendable establecer medidas para controlar la emisión de dichos contaminantes a la atmósfera, ya que esta ciudad tiene características propicias para la concentración de los mismos.

Las bases fisiológicas que pudieran explicar el aumento de las crisis de asma, durante los días en que se incrementan los niveles de contaminantes, son que tanto el ozono como las partículas actúan como irritantes del tracto respiratorio, lo que activa ciertos reflejos del sistema de inervación del mismo, produciendo reacciones no inflamatorias y una hiperreactividad bronquial. ${ }^{31,32}$ Los episodios agudos comienzan con el espasmo bronquial, el que ocurre alrededor de media hora después del estímulo y dura de tres a cuatro horas; esta es la fase llamada precoz. La mayoría de los casos tienen una fase retardada o tardía que se presenta de seis a ocho horas después, cuando aparece la inflamación y de nuevo el broncoespasmo, lo que puede durar horas, días o semanas; a medida que el padecimiento se hace más grave o crónico, la inflamación predomina sobre el espasmo. ${ }^{30,31}$

El hecho de que pueda existir una fase tardía, de días o semanas en la que los síntomas se acentúan, pudiera explicar el efecto en las consultas a urgencias encontrado días después de que aumentan los niveles de los contaminantes, ${ }^{33}$ aunado al patrón de uso de los servicios de salud de la población que muchas veces no es inmediato.

Pudiera existir una interacción entre el ozono y las partículas cuando hay una exposición simultánea e incluso con otros contaminantes que no se incluyeron en este estudio. En el caso que aquí se presenta se encontró que las visitas a urgencias aumentan en $1 \%$ cuando los niveles de contaminantes se incrementan en 20 unidades simultáneamente y en el caso de $\mathrm{PM}_{10}$ su efecto se incrementa hasta $5.03 \%$ más cuando los niveles de ozono se ubican por arriba de 49 ppb (mediana de $\mathrm{O}_{3}$ en el periodo de estudio). Algunos estudios han señalado que existen efectos sinérgicos entre estos contaminantes y algunas enfermedades respiratorias. Téllez-Rojo y colaboradores ${ }^{34}$ informan un importante efecto de $\mathrm{PM}_{10}$ sobre el número total de muertes por enfermedad respiratoria en ancianos, 
cuando los niveles de $\mathrm{O}_{3}$ en el ambiente rebasan 116 ppb.

En la figura 1 se muestra el suavizamiento de las consultas por asma estimadas en relación con el nivel promedio de $\mathrm{PM}_{10}$ durante siete días consecutivos. Esta gráfica muestra un punto de inflexión aproximado a los $40 \mu \mathrm{g} / \mathrm{m}^{3}$, lo que parece indicar que, hasta antes de este nivel, el efecto de las partículas sobre las consultas por asma es constante y que cuando se rebasa se presenta una franca asociación positiva. Al contrario de lo que sucede con la temperatura, cuando ésta rebasa los $15^{\circ} \mathrm{C}$ las consultas por asma tienden a disminuir.

Algunas limitaciones de este estudio son que los datos de salud se obtuvieron de manera retrospectiva por lo que no se pudieron validar los diagnósticos, pero debido a que provienen de una misma institución (IMSS) pensamos que dichos diagnósticos fueron aplicados utilizando criterios similares. Los resultados no pueden ser extrapolables a toda la población, ya que el estudio se realizó únicamente con los datos disponibles en hospitales del IMSS y no toda la población recurre a estos servicios; además, sólo se analizaron las consultas de urgencias como un evento centinela, pues probablemente son representantes de los casos más graves. Sin embargo, es probable que algunos casos de urgencia por problemas respiratorios hayan acudido a solicitar atención a servicios de consulta externa de la misma institución o de otras instituciones médicas, ocasionando una subestimación en el núme-

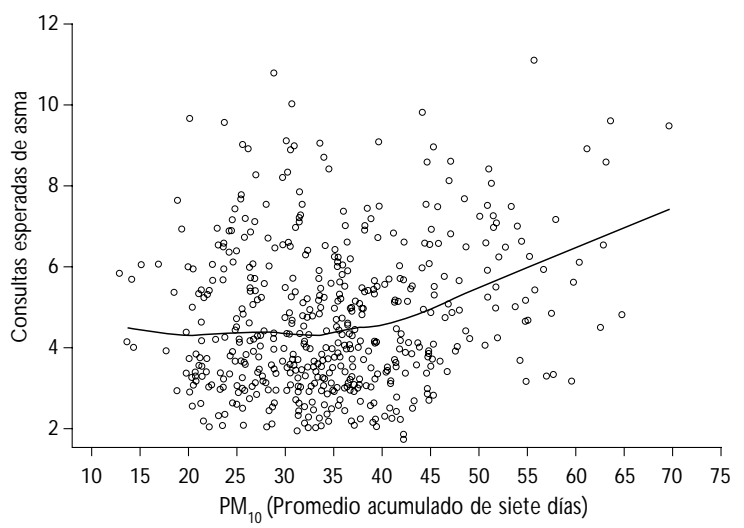

Figura 1. Suavizamiento de las Consultas esperadas DE ASMA DADOS EL PROMEDIO ACUMULADO DE SIETE DÍAS de niveles de PM $_{10}$. Ciudad Juárez, Chihuahua, de JULIO DE 1997 A DICIEMBRE DE 1998 ro de casos; sin embargo, es poco probable que esta subestimación esté relacionada con la variación diaria de los niveles de contaminación atmosférica, en consecuencia, la magnitud real de la asociación estaría subestimada. Asimismo, se observó que los errores de captura detectados se distribuyeron de forma aleatoria durante el periodo de estudio, por lo que no muestran algún patrón asociado con los niveles diarios de exposición, lo que llevaría al efecto estimado hacia el valor nulo.

Otra de las limitaciones es inherente al propio diseño. En los estudios ecológicos no se cuenta con evaluación o medición individual sino a nivel poblacional de la exposición, de tal forma que se asume que la exposición se distribuyó homogéneamente en todo el grupo, lo que se pudiera corregir utilizando monitores intra y extramuros para una mejor medición de la exposición.

Este estudio es el primero que se realiza en una ciudad de la República mexicana diferente de la ciudad de México y que está expuesta a niveles relativamente bajos de contaminación; asimismo, es el primero que analiza la relación entre contaminación atmosférica y enfermedades respiratorias con metodología de series de tiempo. Uno de los hallazgos más importantes de este trabajo es la asociación observada a niveles por debajo de la norma (cuadro II). Es preciso señalar, además, que este estudio es consistente con los trabajos análogos realizados en otras ciudades del mundo (cuadro I), en los que las mediciones de los contaminantes son similares a las encontradas en Ciudad Juárez y están muy por debajo de los registrados en lugares como la ciudad de México. Por este motivo, la vigilancia sistemática aportaría elementos para una racionalización de las normas.

Instituciones como el IMSS capturan información muy valiosa para la vigilancia de la salud de la población, por lo que es muy importante mantener y tecnificar la elaboración permanente de estas bases de datos con el fin de constituir un sistema real y permanente de vigilancia epidemiológica, y evaluar el efecto de factores ambientales como los contaminantes del aire, sobre la salud y el implemento de programas de control.

\section{Agradecimientos}

Agradecemos la colaboración del doctor James Vanderslice, de la Universidad de El Paso, Texas, por su colaboración en la obtención de información sobre niveles de contaminantes, así como su apoyo incondicional en este proyecto; asimismo, al Instituto Mexica- 
no del Seguro Social, en especial a la doctora Margarita Levario, por las facilidades brindadas en la obtención de los registros de salud.

\section{Referencias}

1. Instituto Nacional de Estadística, Geografía e Informática. Cuaderno estadístico municipal, Ciudad Juárez, Estado de Chihuahua.A guascalientes (Ags.): IN EGI, 1996.

2. Dirección General de Gestión e Información Ambiental, Instituto Nacional de Ecología. Programa de G estión de la Calidad del Aire de Ciudad Juárez 1998-2002. México, D.F.: Secretaría de Salud, Secretaría de Medio Ambiente, Recursos N aturales, A gua y Pesca, 1998:28-32.

3. Castillejos M, Gold DR, D ockery DW, Tosteson T, Baum T, Speizer FE. Effects of ambient ozone on respiratory function and symptoms in Mexico City schoolchildren. Am Rev Respir Dis 1992;145:276-282.

4. Bascom R, Bromberg PA, Costa DA. Health effects of outdoor air pollution. Am J Respir Crit Care Med 1996;153(1):3-50.

5. Romieu I, Cortés LM, Velasco SR, Sánchez S, Meneses F, HernándezAvila M.Air pollution and school absenteeism among children in Mexico City. Am J Epidemiol 1992;135(12):1524-1531.

6. Schwartz J, Slater D, Larson TV, Pierson W E, Koening JQ . Particulate air pollution and hospital emergency room visits for asthma in Seattle. Am Rev Respir Dis 1993;147:826-831.

7. Romieu I, Meneses F, Sienra-Monge J, Huerta J, Ruiz-Velazco S, W hite MC et al. Effects of urban air pollutants on emergency visits for childhood asthma in Mexico City.Am J Epidemiol 1995;141(6):546-553.

8. Moolgavkar SH, Luebeck EG, Anderson EL. Air pollution and hospital admission for respiratory causes in Minneapolis, St. Paul and Birmingham. Epidemiology 1997:8(4):364-370.

9. Thurston GD, Ito K, Kinney PL, Lippmann M. A multi-year study of air pollution and respiratory hospital admissions in three $\mathrm{N}$ ew York state metropolitan areas: Result for 1988 and 1989 summers. J Exp Anal Environ Epidemiol 1992; 2(4):429-450.

10. Bates DV, Sitzo R. Air pollution and hospital admissions in Southern O ntario:The acid summer haze effect. Environ Res 1987;43:317-331.

11.W hite M, Etzel R,W allace D,W ilcox W, Lloyd C. Exacerbation of childhood asthma and ozone pollution in Atlanta. Environ Res 1994;65:56-68. 12. Tenias JM, Ballester F, Rivera ML. Association between hospital emergency visits for asthma and air pollution in Valencia, Spain. 0 ccup Environ Med 1998; 55(8):541-547.

13. Lacasaña-N avarro M, A guilar-Garduño C, Romieu I. Evolución de la contaminación del aire e impacto de los programas de control de tres megaciudades de América Latina. Salud Publica Mex 1999;41(3):203-215. 14. Sunyer J,Antón JM, Murillo C, Saez M. Effects of urban air pollution on emergency room admissions for chronic obstructive pulmonary disease. Am J Epidemiol 1991;134(3):277-286.

15.Atkinson RW, Anderson HR, Strachan D P, Bland JM, Bremner SA, Ponce de Leon A. Short-term association between outdoor air pollution and visits to accidents and emergency departments in London for respiratory complaints. Eur Respir J 1999; 13(2):257-265.
16. D elfino RJ, Murphy-Multon AM, Becklake MR. Emergency room visits for respiratory illness among the elderly in Montreal: Association with low level ozone exposure. Environ Res 1998; 76(2):67-77.

17. Clifford PW, Ronald PC, Paul JL. Relation between summertime ambient ozone levels and emergency department visits for asthma in central $\mathrm{N}$ ew Jersey. Environ Health Perspect 1995; 103(suppl 2):97-102.

18. Stieb DM, Burnett RT, Beveridge RC, Brook JR. Association between ozone and asthma emergency department visits in Saint John, N ew Brunswick, C anada. Environ Health Perspect 1996;104(12):1354-1360.

19. Lipsett M, Hurley S, O stro B. Air pollution and emergency room for asthma in Santa Clara County, California. Environ Health Perspect 1997;105(2):216-222.

20.Téllez-Rojo MM, Romieu I, Peña M, Ruiz-Velasco S, Meneses F, H ernández-A vila M. Efecto de la contaminación ambiental sobre las consultas por infecciones respiratorias en niños de la ciudad de México. Salud Publica Mex 1998:39:513-520.

21. 0 rganización Panamericana de la Salud/0 rganización Mundial de la Salud. Clasificación Internacional de Enfermedades ga Rev. Modificación Clínica, $4^{a}$ ed.W ashington, D.C.: O PS/O MS, 1975.

22. O rganización Panamericana de la Salud/O rganización Mundial de la Salud. Clasificación Internacional de Enfermedades $10^{a}$ Rev. Modificación Clínica, $5^{a}$ ed.W ashington, D.C.: O PS/O MS, 1995.

23. Peter J, D iggle, Kung-Yee L, Z eger S.A nalysis of longitudinal data. $0 \mathrm{x}$ ford: Science O bligations, 1995.

24. Schwartz J, Spix C, Touloumi G, Bacharova L, Barumamdzadeh T, Tertre $U$. Methodological issues in studies of air pollution and daily counts of deaths or hospital admissions. J Epidemiol Community Health 1996;50 (suppl 1):3-11.

25. Hastie T, T ibshiran R. Generalized additive models. Condon: C hapman and Hill, 1990.

26. Illabaca M, O laeta I, Campos E, Téllez-Rojo M, Romieu I. Association between levels of fine particle and emergency visits for pneumonia and other respiratory illnesses among children in Santiago, C hile. J Air W aste Manage Assoc 1999;49:154-163.

27. Montealegre $F, C$ ahrdon $D$, Tarats $H$. Enviromental factors precipiting bronchiat asthma exacerbations in Southern Puerto Rico:A pilot study. J Asthma 1993;30(3):219-227.

28. MCC onell R, Berhane K, Gulliand F, London SJ,Vora H,Avol E et al. Air pollution and bronchitis symptoms in Southern California. Environ Health Perspect 1999;107(9):757-769.

29. Goren A, Hellman S, Gabbay Y, Brenner S. Respiratory problems associated with exposure airborne particles in the community. Arch Environ Health 1999;54(3):165-171.

30. Borja-A burto VH, Castillejos M, Gold DR, Bierzwinski S, Loomis D. Mortality and ambient of fine particles So uthwest Mexico City, 1993-1995. Environ Health Perspect 1998;849-855.

31. Committee on Environmental Health. Ambient air pollution: Respiratory hazards to children. Pediatrics 1993;91:1210-1213.

32. Bellanti JA. Patogénesis del asma. Rev Alerg Mex 1994;2:39-41.

33.Vázquez F, G ovea C, Beltrán F,Torres I.A nálisis de la demanda de atención del niño asmático en el servicio de urgencias.Alergia 1994;(3):80-83. 34.Téllez-Rojo MM, Romieu I,Velasco S. Daily respiratory mortality and pollution in Mexico City:The importance of considering place of death. Aceptado para su publicación en Eur Resp Journal. 